\title{
Full Rough Sets
}

\author{
Ray-Ming Chen \\ Department of Computer Science \\ Friedrich-Alexander-Universität Erlangen-Nürnberg \\ Martenstraße 3, 91058 Erlangen, Germany \\ ray.chen@fau.de
}

\begin{abstract}
I will analyze Pawlak's rough sets and extend the usual setting of characterization via an equivalence relation to any arbitrary relation, or a full relation. I extract the specification of rough sets by two operators: probable operator and sure operator. This would provide a general framework to analyze full rough sets and to compare them with Pawlak's rough sets. It also facilitates all the computation and further expansion. This paper extends Pawlak's approximation approach to any arbitrary relations and explicitly defines several computational operators to study the closeness regarding the characterization of operations on sets.
\end{abstract}

\section{INTRODUCTION}

I N PAWLAK'S paper "Rough Sets", he studied the properties of an approximation space $A=(U, R)$, where $U$ is a universe and $R$ is an equivalence relation. He then in Section 2.3 (pp. 344-345), listed 30 identities of the properties of the approximation space and these become a foundation of his theory. These identities specify the properties of set operations and best upper approximation $\overline{A p r}$ and best lower approximation $A p r$. These identities also have some practical applications. For example, to calculate the best upper and lower approximation of the $X \cup Y \subseteq U$, one only needs to calculate the respective best approximations of $X$ and $Y$. This would save some resources and calculations if it is put into practical applications. When I read Pawlak's "Rough Sets", the first question comes to my mind is: Could I extend this equivalent relation to any arbitrary relation? Then the second question follows: if I extend it to any arbitrary relation, would these identities hold? Then it goes to the third question: under which circumstances could these identities hold? And then the last question: is there any relationship between different set operations under these approximations?

Such extension is also briefly mentioned in several occasions, for example, a chapter written by Hung Son Nguyen and Andrzej Skowron [7]. Indeed, there are some authors trying to generalize and extend $R$, in particular, Y.Y.Yao ([3][4],[5],[6]). In Yao's papers, he specifically defines an extended $R$, say $R^{*}$ (a non-equivalence relation), and then studies the properties of best approximations and these generalized rough sets.

Unlike Yao's papers, my intention is not to specify or study any particular extended relation. My intention is to study any arbitrary relations. Another main difference is my research will focus on answering my last two questions, which are rarely mentioned in any other papers, not even in Pawlak's original paper. The results will be presented in Section VI. Another characteristic of my paper is to automate full rough sets approximation, in the sense that a machine or a computer can easily implement the calculations. In order to achieve this, in Section III,I introduce several operators and a characteristic matrix to facilitate the whole computation. Throughout the whole paper, I also compare the differences between Pawlak's rough sets and full rough sets, in particular, Example 18.

\section{BACKGROUND}

In the typical characterization of a crisp target set, an equivalence relation is used to partition the universe and then the partition is served as a classifier for the target sets. However, in the real world, one needs to develop a much more complicated classifier for the target sets. To begin with, let me show some cases that would involve some complicated relations to form granule knowledge. In reality, there might exist more than one equivalence relation and no favorite is made or the interpretation of data needs some tolerance or other reasons. Then one has to contrive a much more complicated classifier.

Example 1 (tolerant classifier). An experimenter observed some viruses' infection rates of cows and recorded them as the following table:

\begin{tabular}{|c|c||c|c|}
\hline Viruses & Infection Rate (\%) & Viruses & Infection Rate (\%) \\
\hline$a$ & 8.22 & $g$ & 40.19 \\
\hline$b$ & 50.21 & $h$ & 11.37 \\
\hline$c$ & 32.49 & $i$ & 20.01 \\
\hline$d$ & 18.41 & $j$ & 10.73 \\
\hline$e$ & 29.83 & $k$ & 19.89 \\
\hline$f$ & 1.24 & $l$ & 9.86 \\
\hline
\end{tabular}

TABLE I

VIRUS INFECTION RATE

Considering some degree of tolerance for the measurements in the endpoints, a biologist forms his granule knowledge of these viruses as follows: a type one (or $T 1$ ) virus is a virus whose infection rate lies between 0 and 10.25; a type two (or T2), 9.75 and 20.25; a type three (or T3), 19.75 and 30.25 ; a type four (or T4), 29.75 and 40.25 and a type five (or $T 5$ ), 39.75 and 50.25 . He then forms the classifier $\{\{a, f, l\},\{d, h, i, j, k, l\},\{e, i, k\},\{c, e, g\},\{b, g\}\}$.

This classifier will be his granule knowledge to characterize target sets. Furthermore, some granule knowledge might be formed subjectively or randomly. 
Example 2 (Relational Tree). Suppose the universe $U=\left\{x_{1}, x_{2}, x_{3}, x_{4}\right\}$ and the following relation or its directed diagram:

$\mathcal{R}:=\left\{\left(x_{1}, x_{1}\right),\left(x_{1}, x_{4}\right),\left(x_{2}, x_{1}\right),\left(x_{2}, x_{2}\right),\left(x_{2}, x_{3}\right),\left(x_{3}, x_{1}\right)\right.$, $\left.\left.\left(x_{3}, x_{2}\right),\left(x_{3}, x_{3}\right),\left(x_{4}, x_{2}\right)\right)\right\}$.

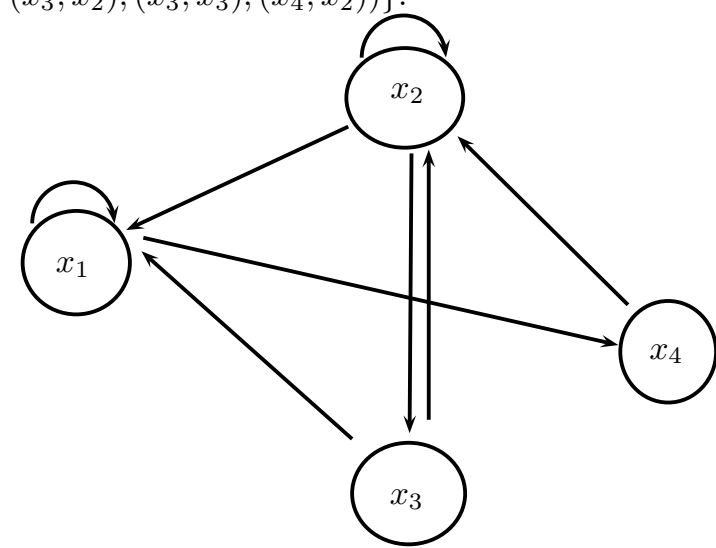

If the granule knowledge is formed via this relation, then the classifier would be $\left\{\left\{x_{1}, x_{4}\right\},\left\{x_{1}, x_{2}, x_{3}\right\},\left\{x_{1}, x_{2}\right\},\left\{x_{2}\right\}\right\}$. This generalized relation is also studied in Section 2.1 of Yao's paper [3] "On Generalizing Rough Set Theory".

In addition, there are many other cases that would involve non-equivalence relations. For example, some data is missing in forming an equivalent relation. In Section III, full rough sets will be introduced. In Section VI, I will study the properties of full rough sets.

\section{PRELIMINARY}

First of all, let us recall how Pawlak defines his rough sets. Let $A=(U, R)$ be an approximation space. He then defines the $R$-lower approximation of $X \subseteq U, \operatorname{Apr}_{A}(X):=$ $\cup_{x \in U}\{R(x): R(x) \subseteq X\}$ and the $R$-upper approximation of $X \subseteq U, \overline{\operatorname{Apr}_{A}}(X):=\cup_{x \in U}\{R(x): R(x) \cap X \neq \emptyset\}$, where $R \subseteq U \times U$ is an indiscernibility relation and $R(x)$ is the equivalence class of $R$ determined by the element $x \in U$. In order to name it, we call the partition (or equivalence classes) $U / R$ a classifier in the sense that any subset $X \subseteq U$ is classified by a pair of rough sets via this partition. Now the first step for me to extract this definition and generalize it to any arbitrary relation is to use a functional classifier. Let $U$ be a finite universe. In this paper, I will assume there exists a bijective function to specify the elements in $U$. Let $\mid U^{\downarrow} \equiv\{1,2, \ldots,|U|\}$.

Definition 1. For any function from $U$ to $\mathcal{P}(U)$, which is chosen as a classifier, is called a functional classifier. Let $\mathcal{K}$ : $U \rightarrow \mathcal{P}(U)$ be a functional classifier.

It is worth mentioning that this definition also appears in Y.Y.Yao's paper [3] in the name of a successor neighborhood $R_{s}(x)=\{y \mid y \in U, x R y\}$, where $R \subseteq$ is any arbitrary binary relation. But we differ in the way forming upper and lower best approximation. Here I reiterate his definitions: $\operatorname{apr}_{R}(A)=$ $\left\{x \mid R_{s}(x) \subseteq A\right\}$ and $\overline{a p r_{R}}(A)=\left\{x \mid R_{s}(x) \cap A \not \emptyset\right\}$. Now I introduce some definitions and notations.
Inspired by Lotfi A. Zadeh's approach (in [8]) using characteristic functions to represent fuzzy sets, I introduce characteristic matrix to represent a functional classifier to facilitate the computations. Each functional classifier $\mathcal{K}$ can then be represented by a matrix $\mathcal{K}^{*}$ in $2^{U \times U}$ via its characteristic values. We name $\mathcal{K}^{*}$ a characteristic classifier.

Example 3. Suppose $U=\left\{u_{1}, u_{2}, u_{3}\right\}$ and $\mathcal{K}\left(u_{1}\right)=$ $\left\{u_{1}, u_{2}, u_{3}\right\}, \mathcal{K}\left(u_{2}\right)=\left\{u_{1}, u_{3}\right\}$ and $\mathcal{K}\left(u_{3}\right)=\left\{u_{2}\right\}$. Then one has the following matrix-operation form:

$$
\mathcal{K}=\left[\begin{array}{l}
\mathcal{K}\left(u_{1}\right) \\
\mathcal{K}\left(u_{2}\right) \\
\mathcal{K}\left(u_{3}\right)
\end{array}\right]=\left[\begin{array}{lll}
1 & 1 & 1 \\
1 & 0 & 1 \\
0 & 1 & 0
\end{array}\right]\left[\begin{array}{l}
u_{1} \\
u_{2} \\
u_{3}
\end{array}\right]
$$

Thus $\mathcal{K}$ can be represented by its characteristic matrix

$$
\mathcal{K}^{*}=\left[\begin{array}{l}
\mathcal{K}^{*}(1) \\
\mathcal{K}^{*}(2) \\
\mathcal{K}^{*}(3)
\end{array}\right]=\left[\begin{array}{lll}
1 & 1 & 1 \\
1 & 0 & 1 \\
0 & 1 & 0
\end{array}\right],
$$

where $\mathcal{K}^{*}(j)$ is the characteristic function of $\mathcal{K}\left(u_{j}\right)$.

By reading at each row vector, one knows immediately the member of that class. This representation would facilitate our computation largely and it could also be programmed to automate the approximation process. In addition, each subset $X \subseteq U$ is also represented by its characteristic function $\rho \in 2^{U}$, for example, a subset $X=\left\{u_{1}, u_{4}\right\}$ in a universe $U$, with $|U|=5$ would be represented by its characteristic function [10010]. Since we restrict $K$ to be a characteristic classifier, and thus $K^{*}$ is always a square matrix with a dimension $|U|$. Of course, one can further lift such restriction and extend $K$ to a relational classifier, i.e., for some element $u \in U$, there associate more than two granule classes $K_{1}(u)$ and $K_{2}(u)$. In this paper, we will not go into such extension.

Definition 2. For any arbitrary subset $\rho \in 2^{U}$, define $\rho(n)$ to be the $n$ 'th element of $U$; for any arbitrary characteristic classifier $T \in 2^{U \times U}$, define $T(n)$ be the $n$ 'th row of $T$ and $T(m, n)$ be the element in $m$ 'th row and $n$ 'th column.

Let $\mathcal{K}^{*}\left(|U|^{\downarrow}\right)=\left\{\mathcal{K}^{*}(j): j \in|U|^{\downarrow}\right\}$, the set of all the granule knowledge (classes). Take Example 3 for example. Then one has $\mathcal{K}^{*}\left(\mid U^{\downarrow}\right)=\left\{\mathcal{K}^{*}(1), \mathcal{K}^{*}(2), \mathcal{K}^{*}(3)\right\}=$ $\left\{\left[\begin{array}{lll}1 & 1 & 1\end{array}\right],\left[\begin{array}{lll}1 & 0 & 1\end{array}\right],\left[\begin{array}{lll}0 & 1 & 0\end{array}\right].\right\}$. We can further define some set union and intersection operations in the form of characteristic functions.

Definition 3. Define $\cup^{*}: 2^{U} \times 2^{U} \rightarrow 2^{U}$ by $\left(\rho \cup^{*} \tau\right)(n):=$ $\sup \{\rho(n), \tau(n)\}$.

Example 4. $\left[\begin{array}{lllll}1 & 0 & 0 & 1 & 1\end{array}\right] \cup^{*}\left[\begin{array}{lllll}0 & 1 & 0 & 1 & 0\end{array}\right]=$ $\left[\begin{array}{lllll}1 & 1 & 0 & 1 & 1\end{array}\right]$.

Definition 4. Define $\cap^{*}: 2^{U} \times 2^{U} \rightarrow 2^{U}$ by $\left(\rho \cap^{*} \tau\right)(n):=$ $\inf \{\rho(n), \tau(n)\}$.

Example 5. $\left[\begin{array}{lllll}1 & 0 & 0 & 1 & 1\end{array}\right] \cap^{*}\left[\begin{array}{lllll}0 & 1 & 0 & 1 & 0\end{array}\right]=$ $\left[\begin{array}{lllll}0 & 0 & 0 & 1 & 0\end{array}\right]$. 
This kind of definitions is common for defining the set operation, for example, in Zadeh's paper [8]. We can further define the subset operation in the form of characteristic functions.

Definition 5. For all $\rho, \tau \in 2^{U}$, define $\rho \subseteq^{*} \tau$ iff $\forall n \in$ $|U|^{\downarrow}[\rho(n) \leq \tau(n)]$.

We also use the notations $\overrightarrow{0}: \mid U^{\downarrow} \rightarrow\{0,1\}$ with $\overrightarrow{0}(n)=0$ for $\forall n \in \mid U^{\downarrow}$ and $\overrightarrow{1}:|U|^{\downarrow} \rightarrow\{0,1\}$ with $\overrightarrow{1}(n)=1$ for $\forall n \in|U|^{\downarrow}$. Let $\rho \in 2^{U}$ be a target set. Now we start to define upper and lower best approximations. In correspondence to our setting, here I name them full upper bound and full lower bound, respectively. The definition of these bounds will be presented in the form of operations over a characteristic classifier. Now we can define the full rough sets as follows:

Definition 6 (Full Upper Bound). Define the full upper bound of $\rho$ as $\rho^{+}=\cup^{*}\left\{\tau \in \mathcal{K}^{*}\left(|U|^{\downarrow}\right): \tau \cap^{*} \rho \neq \overrightarrow{0}\right\}$.

Definition 7 (Full Lower Bound). Define the full lower bound of $\rho$ as $\rho^{-}=\cup^{*}\left\{\tau \in \mathcal{K}^{*}\left(\mid U^{\downarrow}\right): \tau \cap^{*} \rho \neq \overrightarrow{0}, \tau \subseteq^{*} \rho\right\}$.

$\cup^{*} S$ denotes the $\cup^{*}$ operation over all the elements in set $S .\left(\rho^{-}, \rho^{+}\right)$are called full rough sets of the characteristic target set $\rho$ via the characteristic classifier $K^{*}$. These full rough sets will be further characterized in Lemma 1 . These definitions indeed are the characteristic version of the following usual rough set definitions (except now we consider arbitrary relation). Let $U$ be an arbitrary universe. Let $X \subseteq U$ be a target set. Let $R \subseteq U \times U$ be an arbitrary relation. Let $R(x)=\{y \in U: x R y\}$. Let the functional classifier $\mathcal{K}=\{R(x): x \in U\}$.

Definition 8 (Full Upper Bound). Define the full upper bound of $X$ as $X^{+}=\bigcup_{x \in U}\{R(x): R(x) \cap X \neq \emptyset\}$.

Definition 9 (Full Lower Bound). Define the full lower bound of $X$ as $X^{-}=\cup_{x \in U}\{R(x): R(x) \cap X \neq \emptyset, R(x) \subseteq X\}$.

Here I change the usual setting of best lower approximation a bit by adding a clause $R(x) \cap X \neq \emptyset$. This change has no impact on the current theory. It is just a condition for set specification when one intends for further extension. We use the notation $\rho^{-1}\{1\}=\left\{n \in|U|^{\downarrow}: \rho(n)=1\right\}$, for all $\rho \in 2^{U}$. $\rho^{-1}\{1\}$ shows the elements in $\rho$. Now I define the difference of two sets in the form of characteristic functions.

Definition 10. Define $-^{*}: 2^{U} \times 2^{U} \rightarrow 2^{U}$ by $\left(\rho-^{*} \tau\right)(n)=$ $\inf \{\rho(n), 1-\inf \{\rho(n), \tau(n)\}\}$.

Example 6. $\left[\begin{array}{lllll}1 & 0 & 0 & 1 & 0\end{array}\right]-{ }^{*}\left[\begin{array}{lllll}0 & 1 & 0 & 1 & 1\end{array}\right]=$ $\left[\begin{array}{lllll}1 & 0 & 0 & 0 & 0\end{array}\right]$.

There are many ways to define a characteristic-function version of a difference set operation. For any set $X \in \mathcal{P}(U)$, let $X^{*} \in 2^{U}$ denote its characteristic function. In order to construct a computational approach for upper or lower best approximations, I extract the definitions and redefine them in the form of characteristic functions. To test whether two sets (characteristic functions) intersects or not, I define the following intersection indicator $\bullet$.
Definition 11 (Intersection Indicator:•). Define $\bullet: 2^{U} \times 2^{U} \rightarrow$ $\{0,1\}$ by $\rho \bullet \tau:=\sup \left\{\tau(n): n \in \rho^{-1}\{1\}\right\}$.

The computational idea for this definition is: to see whether $\tau$ and $\rho$ has common elements or not, one finds the order numbers of $\rho$ and then checks whether these numbers are shared by $\tau$. If yes, then they intersects with each other; if not, there is no intersection between them, i.e., the intersection is $\overrightarrow{0}$ (the characteristic function for the empty set). In sum, if $\rho \bullet \tau=1$, it means $\rho$ intersects $\tau$; if $\rho \bullet \tau=0$, it means $\rho$ and $\tau$ are disjointed.

Example 7. $\left[\begin{array}{lllll}1 & 1 & 0 & 1 & 1\end{array}\right] \bullet\left[\begin{array}{lllll}0 & 1 & 1 & 1 & 0\end{array}\right]=1$.

Now we almost go into the main body of my approach. Recall a usual setting of a best approximation, for example, $\underline{\operatorname{Apr}_{A}}(X):=\cup_{x \in U}\{R(x): R(x) \subseteq X\}$. In order to put it into a computational content, I separate it into two parts: specification test and value assigned. In this case, the specification to be tested is: $R(x) \subseteq X$. So one tests whether $R(x) \subseteq X$ or not. If yes, then one assigns one value for the $\operatorname{Apr}_{A}(X)$ and if no, one assigns the other value. There are two main reasons for such approach: firstly, to automate the computation and secondly, to leave a room for further definition of the usual $\cup$ approximation-indeed one would be given much more freedom to assign the approximation values.

In the next claim, I will convert the usual specification of rough sets into the context of characteristic functions and classifiers.

Claim 1. Let $Y \in \mathcal{P}(U)$ be arbitrary. Let $\mathcal{K}$ be an arbitrary classifier. Then

1) $Y \cap \mathcal{K}\left(u_{j}\right) \neq \emptyset$ iff $Y^{*} \cap^{*} \mathcal{K}^{*}(j) \neq \overrightarrow{0}$ iff $Y^{*} \bullet \mathcal{K}^{*}(j)=1$.

2) $\mathcal{K}\left(u_{j}\right) \subseteq Y$ iff $\mathcal{K}^{*}(j) \subseteq \subseteq^{*} Y^{*}$ iff $\left(\overrightarrow{1}-{ }^{*} Y^{*}\right) \bullet \mathcal{K}^{*}(j)=0$.

3) $Y \cap \mathcal{K}\left(u_{j}\right) \neq \emptyset$ and $\mathcal{K}\left(u_{j}\right) \subseteq Y$ iff $Y^{*} \cap^{*} \mathcal{K}^{*}(j) \neq \overrightarrow{0}$ and $\mathcal{K}^{*}(j) \subseteq \subseteq^{*} Y^{*}$ iff inf $\left\{Y^{*} \bullet \mathcal{K}^{*}(j), 1-\left(\overrightarrow{1}-{ }^{*} Y^{*}\right) \bullet\right.$ $\left.\mathcal{K}^{*}(j)\right\}=1$ iff $Y^{*} \bullet \mathcal{K}^{*}(j)-\left(\overrightarrow{1}-{ }^{*} Y^{*}\right) \bullet \mathcal{K}^{*}(j)=1$

Proof. (1) and (2) follow immediately from the definitions. Here I show (3): inf $\left\{Y^{*} \bullet \mathcal{K}^{*}(j), 1-\left(\overrightarrow{1}-{ }^{*} Y^{*}\right) \bullet \mathcal{K}^{*}(j)\right\}=1$ iff $\left.Y^{*} \bullet \mathcal{K}^{*}(j)=1-\left(\overrightarrow{1}-{ }^{*} Y^{*}\right) \bullet \mathcal{K}^{*}(j)\right\}=1$ iff $Y^{*} \bullet \mathcal{K}^{*}(j)=1$ and $\left(\overrightarrow{1}-{ }^{*} Y^{*}\right) \bullet \mathcal{K}^{*}(j)=0$ iff $Y^{*} \cap^{*} \mathcal{K}^{*}(j) \neq \overrightarrow{0}$ and $\mathcal{K}^{*}(j) \subseteq^{*}$ $Y^{*}$.

This claim indeed forms a cornerstone which converts the usual rough sets formation into a computational characteristic target sets and characteristic classifier. From this claim, we characterize the set specification $Y \cap \mathcal{K}\left(u_{j}\right) \neq \emptyset$ with a probable operator po defined in Definition 13. We can also characterize the set specification $Y \cap \mathcal{K}\left(u_{j}\right) \neq \emptyset$ and $\mathcal{K}\left(u_{j}\right) \subseteq$ $Y$ with a sure operator so defined in Definition 14. Since we have already defined an intersection indicator, we could now further define an intersection operator $\odot$ to compute and test the intersection between a target set (a characteristic function) and a functional classifier (a characteristic classifier).

Definition 12 (Intersection Operator $\odot$ ). Define $\odot: 2^{U} \times$ $2^{U \times U} \rightarrow 2^{U}$ by $(\rho \odot T)(n):=\rho \bullet T(n)$. 
Example 8. $\left[\begin{array}{lllll}1 & 0 & 1 & 0 & 0\end{array}\right] \odot\left[\begin{array}{lllll}1 & 0 & 1 & 0 & 0 \\ 0 & 1 & 1 & 0 & 0 \\ 0 & 0 & 0 & 0 & 1 \\ 0 & 1 & 0 & 0 & 0 \\ 1 & 1 & 1 & 0 & 1\end{array}\right]=$

This operator shows whether the target set intersects with the classifier or not. With this operator, one can now easily visualize the intersection between target sets and its classifier.

Now we separate the set specifications from the set comprehension via two operators: specification operators and value operators. Specification operators consist of two operators: probable operator $(p o)$ and sure operator $(s o)$.

Definition 13. Define $p o: 2^{U} \times 2^{U \times U} \rightarrow 2^{U}$ by $p o(\rho, T):=$ $\rho \odot T$

Definition 14. so $: 2^{U} \times 2^{U \times U} \rightarrow 2^{U}$ by $s o(\rho, T)=\rho \odot T-^{*}$ $\left(\overrightarrow{1}-{ }^{*} \rho\right) \odot T$.

Example 9. $s o\left(\left[\begin{array}{lllll}1 & 0 & 0 & 1 & 1\end{array}\right],\left[\begin{array}{lllll}1 & 0 & 0 & 0 & 1 \\ 0 & 1 & 1 & 0 & 0 \\ 1 & 0 & 0 & 1 & 0 \\ 0 & 1 & 1 & 1 & 0 \\ 1 & 0 & 1 & 0 & 1\end{array}\right]\right)=$ $\left[\begin{array}{lllll}1 & 0 & 1 & 1 & 1\end{array}\right]-{ }^{*}\left[\begin{array}{lllll}0 & 1 & 0 & 1 & 1\end{array}\right]=\left[\begin{array}{lllll}1 & 0 & 1 & 0 & 0\end{array}\right]$

Definition 15. We say $(\rho, T)$ is a crisp specification iff $p o(\rho, T)=s o(\rho, T)$ and $(\rho, T)$ is a rough specification iff $p o(\rho, T) \neq s o(\rho, T)$.

Though I separate specification and value assignment, in this paper, I will not change the usual setting of $U$-value assignment. The following definitions maintain the usual $\cup$ value assignment in the computational context. In the following definition, we start to specify the form of a classifier.

Definition 16. Define sup ${ }^{*}: 2^{U \times U} \rightarrow 2^{U}$ by $\left(\sup ^{*}(T)\right)(n):=\sup \left\{T(n, m): m \in \mid U^{\downarrow}\right\}$.

Example 10. $\sup ^{*}\left(\left[\begin{array}{llll}0 & 0 & 1 & 1 \\ 0 & 1 & 0 & 0 \\ 0 & 0 & 1 & 0 \\ 0 & 1 & 1 & 1\end{array}\right]\right)=\left[\begin{array}{llll}1 & 1 & 1 & 1\end{array}\right]$.

Example 11. $\sup ^{*}\left(\left[\begin{array}{llll}0 & 0 & 0 & 0 \\ 0 & 1 & 0 & 1 \\ 1 & 0 & 1 & 1 \\ 0 & 0 & 0 & 0\end{array}\right]\right)=\left[\begin{array}{llll}0 & 1 & 1 & 0\end{array}\right]$.

We say that a characteristic classifier $T$ is degenerated if and only if there exists an element 0 in $\sup ^{*}(T)$ and nondegenerated iff all the elements are 1 in $\sup ^{*}(T)$. For example, $T$ in Example 10 is non-degenerated, while in Example 11 is degenerated. For a general setting, one would like to specify the exact form of $T$, for example, $T$ is formed via equivalence relation or other relations. However, I study a more general relation, i.e., a non-generated $T$.

In most of the settings and derivations, the universe $U$ itself is taken as closed under set operations. In order to study the detailed properties, I generalize it to study any subsets of $U$ that are closed under set operations. On the one hand, it helps us to understand that some properties not only belong to the universe per se, but it also applies to its subsets; on the other hand, it also helps us classify a set of target sets that are closed under set operations.

Definition 17. $S \subseteq 2^{U}$ is $\cup^{*}$-closed iff for all $\rho, \tau \in S$, one has $\rho \cup^{*} \tau \in S$.

Definition 18. $S \subseteq 2^{U}$ is $\cap^{*}$-closed iff for all $\rho, \tau \in S$, one has $\rho \cap^{*} \tau \in S$.

Definition 19. $S \subseteq 2^{U}$ is $c^{*}$-closed iff for all $\rho \in S$, one has $\overrightarrow{1}-{ }^{*} \rho \in S$.

Example 12. $S=\left\{\left[\begin{array}{lll}1 & 0 & 1\end{array}\right],\left[\begin{array}{lll}1 & 0 & 0\end{array}\right],\left[\begin{array}{lll}1 & 1 & 1\end{array}\right]\right\}$ is $\cup^{*}$-closed and $\cap^{*}$-closed, but not $c^{*}$-closed.

\section{BASIC IDENTITY}

Let $\rho, \tau, \eta, \theta \in 2^{U}$ be arbitrary. Then we have the following basic identities of set operations in the form of characteristic functions. These identities will be applied in Section VI without an explicit mention.

Claim 2. 1) $\rho \cup^{*}\left(\tau-{ }^{*} \eta\right)=\left(\rho \cup^{*} \tau\right)-^{*}\left(\eta-^{*} \rho\right)$.

2) $\rho-^{*}\left(\tau-{ }^{*} \eta\right)=\left(\rho \cap^{*} \eta\right) \cup^{*}\left(\rho-^{*} \tau\right)$.

3) $\left(\rho-^{*} \tau\right)-^{*} \eta=\rho-^{*}\left(\tau \cup^{*} \eta\right)$.

4) $\rho \cap^{*}\left(\tau-{ }^{*} \eta\right)=\left(\rho \cap^{*} \tau\right)-^{*} \eta=\tau \cap^{*}\left(\rho-^{*} \eta\right)$.

5) $\rho-^{*}\left(\tau \cup{ }^{*} \eta\right)=\left(\rho-{ }^{*} \tau\right) \cap^{*}\left(\rho-{ }^{*} \eta\right)$.

6) $\rho-^{*}\left(\tau \cap^{*} \eta\right)=\left(\rho-{ }^{*} \tau\right) \cup^{*}\left(\rho-^{*} \eta\right)$.

7) $\left(\rho \cup^{*} \tau\right)-^{*} \eta=\left(\rho-{ }^{*} \eta\right) \cup^{*}\left(\tau-{ }^{*} \eta\right)$.

8) $\left(\rho-{ }^{*} \tau\right) \cup^{*} \eta=\left(\rho \cup^{*} \eta\right)-{ }^{*}\left(\tau-{ }^{*} \eta\right)$.

Proof. All these identities follow immediately from the basic set operations in terms of characteristic functions. Take (6) for example. It comes directly from the identity: $A-(B \cap C)=$ $(A-B) \cup(A-C)$ for any arbitrary sets $A, B$ and $C$.

\section{PROPERTY}

First of all, a computational characterization of a target set is derived. Secondly, the properties of all the operators defined will be studied. In the next lemma, I will show how to characterize full rough sets defined in Definition 6 and 7 by the following lemma.

Lemma 1. Given a target set $\rho \in 2^{U}$ and a characteristic classifier $T \in 2^{U \times U}$, one gets its full upper bound (i.e., best upper approximation) $\rho^{+}=p o(\rho, T) \odot T^{t}$ and its full lower bound (i.e., best lower approximation) as $\rho^{-}=\operatorname{so}(\rho, T) \odot T^{t}$, where $T^{t}$ denotes the transpose of the matrix $T$. 
Proof. Suppose $p o(\rho, T)=f$. Then the full upper bound $\rho^{+}=\cup^{*}\left\{T(n): n \in f^{-1}\{1\}\right\}=f \odot T^{t}$. Similarly, let $s o(\rho, T)=g$. Then the full lower bound $\rho^{-}=\cup^{*}\{T(n)$ : $\left.n \in g^{-1}\{1\}\right\}=g \odot T^{t}$.

This lemma shows directly how to compute best upper approximation and best lower approximation. Indeed one only needs to find out the $p o(\rho, T)$ for each target set $\rho$ to get the final result. Henceforth, in this paper, I will focus on the study of the properties of specification operators $p o$ and $s o$.

Definition 20 (Full Upper Bound). Define $u b: 2^{U} \times 2^{U \times U} \rightarrow$ $2^{U}$ to be $u b(\rho, T):=p o(\rho, T) \odot T^{t}$, where $T^{t}$ denotes the transpose of the matrix of $T$.

Definition 21 (Full Lower Bound). Define $l b: 2^{U} \times 2^{U \times U} \rightarrow$ $2^{U}$ to be $l b(\rho, T):=s o(\rho, T) \odot T^{t}$, where $T^{t}$ denotes the transpose of the matrix of $T$.

Example 13. Let the target set be $X=\left\{x_{2}, x_{3}\right\}$. Let $T$ be the characteristic classifier induced by the classifier in Example 2. Let us use this formalism to compute its full rough sets. To begin with, let me compute the usual setting first via Definition 8 and 9. Then one has the best lower bound $X^{-}=\left\{x_{2}\right\}$ and the best upper bound $X^{+}=\left\{x_{1}, x_{2}, x_{3}\right\}$. Now let us look into the characteristic version. The $\mathcal{R}$ induced characteristic classifier is $T=\left[\begin{array}{llll}1 & 0 & 0 & 1 \\ 1 & 1 & 1 & 0 \\ 1 & 1 & 0 & 0 \\ 0 & 1 & 0 & 0\end{array}\right]$ and the characteristic target set is $X^{*}=\left[\begin{array}{llll}0 & 1 & 1 & 0\end{array}\right] . \operatorname{so}\left(X^{*}, T\right)=$ $X^{*} \odot T-{ }^{*}\left(\overrightarrow{1}-{ }^{*} X^{*}\right) \odot T=\left[\begin{array}{llll}0 & 1 & 1 & 1\end{array}\right]-^{*}\left[\begin{array}{llll}1 & 1 & 1 & 0\end{array}\right]=$ $\left[\begin{array}{llll}0 & 0 & 0 & 1\end{array}\right]$. Now we have $l b\left(X^{*}, T\right)=\operatorname{so}\left(X^{*}, T\right) \odot T^{t}=$ $\left[\begin{array}{llll}0 & 0 & 0 & 1\end{array}\right] \odot\left[\begin{array}{llll}1 & 1 & 1 & 0 \\ 0 & 1 & 1 & 1 \\ 0 & 1 & 0 & 0 \\ 1 & 0 & 0 & 0\end{array}\right]=\left[\begin{array}{llll}0 & 1 & 0 & 0\end{array}\right]$; similarly, one has $p o\left(X^{*}, T\right)=\left[\begin{array}{llll}0 & 1 & 1 & 1\end{array}\right]$ and thus $u b\left(X^{*}, T\right)=$ $p o\left(X^{*}, T\right) \odot T^{t}=\left[\begin{array}{llll}1 & 1 & 1 & 0\end{array}\right]$.

Image that the size of the universe is two million and the size of the target set is one million. Are we still using our naked eyes to classify this target set? Basically, this characteristic version provides a systematic way to assign full rough sets to a target set and it also provides a theoretical framework for better understanding of full rough sets.

Once one gets the values of $p o(\rho, T)$ and $s o(\rho, T)$, he gets the values of upper bound and lower bound straightaway. Henceforth, we will focus on studying the properties of $p o$ and $s o$ in this paper. To further understand the above-mentioned operators, I derive some of their properties here. Some of the properties would be applied later. There operators also play vital roles in gaining our main results in Section VI. Let $\rho, \rho^{\prime}, \tau, \tau^{\prime}, \eta \in 2^{U}$ and $T \in 2^{U \times U}$ be arbitrary.

\section{A. Operator •}

Claim 3. If $\rho \subseteq^{*} \tau$ and $\rho^{\prime} \subseteq^{*} \tau^{\prime}$, then $\rho \bullet \rho^{\prime} \leq \tau \bullet \tau^{\prime}$.
Proof. $\rho \bullet \rho^{\prime}=\sup \left\{\rho^{\prime}(n): n \in \rho^{-1}\{1\}\right\} \leq \sup \left\{\rho^{\prime}(n): n \in\right.$ $\left.\tau^{-1}\{1\}\right\} \leq \sup \left\{\tau^{\prime}(n): n \in \tau^{-1}\{1\}\right\}=\tau \bullet \tau^{\prime}$.

Claim 4. $\rho \bullet \tau=\tau \bullet \rho$

Proof. $\rho \bullet \tau=1$ iff there exists $n \in|U|^{\downarrow}$ such that $\rho(n)=$ $\tau(n)=1$.

Claim 5. $\left(\rho \cup^{*} \tau\right) \bullet \eta=\sup \{\rho \bullet \eta, \tau \bullet \eta\}$.

Proof. $\left(\rho \cup^{*} \tau\right) \bullet \eta=\sup \left\{\eta(n): n \in\left(\rho \cup^{*} \tau\right)^{-1}\{1\}=\right.$ $\sup \left\{\eta(n): n \in \rho^{-1}\{1\} \cup \tau^{-1}\{1\}\right\}=\sup \{\sup \{\eta(n): n \in$ $\left.\left.\rho^{-1}\{1\}\right\}, \sup \left\{\eta(n): n \in \tau^{-1}\{1\}\right\}\right\}$.

\section{B. Operator $\odot$}

Let $\rho, \rho^{\prime}, \tau \in 2^{U}$ and $T \in 2^{U \times U}$ be arbitrary.

Claim 6. $\left(\rho \cup^{*} \tau\right) \odot T=(\rho \odot T) \cup^{*}(\tau \odot T)$, i.e., $p o\left(\rho \cup^{*} \tau, T\right)=$ $p o(\rho, T) \cup^{*} p o(\tau, T)$.

Proof. $\left(\left(\rho \cup^{*} \tau\right) \odot T\right)(n)=\left(\rho \cup^{*} \tau\right) \bullet T(n)=\sup \{\rho \bullet T(n), \tau \bullet$ $T(n)\}$, by Claim 5 .

We can then apply this identity to show other special cases.

Example 14. Property (6) and (15) at Section 2.3 in Pawlak's paper [1] are the direct instances of this claim (via Lemma 1). Take property (6) for example: $u b\left(X^{*} \cup^{*} Y^{*}, T\right)=p o\left(X^{*} \cup^{*}\right.$ $\left.Y^{*}, T\right) \odot T^{t}=\left(p o\left(X^{*}, T\right) \cup^{*} p o\left(Y^{*}, T\right)\right) \odot T^{t}=\left(p o\left(X^{*}, T\right) \odot\right.$ $\left.T^{t}\right) \cup^{*}\left(p o\left(X^{*}, T\right) \odot\right) T^{t}=u b\left(X^{*}, T\right) \cup^{*} u b\left(Y^{*}, T\right)$, where $T$ is the characteristic classifier induced by the equivalence relation.

Remark 1. Generally speaking, $\left(\rho \cap^{*} \tau\right) \odot T \neq(\rho \odot$ $T) \cap^{*}(\tau \odot T)$, for example, $\rho=\left[\begin{array}{llll}0 & 1 & 0 & 0\end{array}\right], \tau=$ $\left[\begin{array}{llll}1 & 0 & 0 & 1\end{array}\right], T=\left[\begin{array}{llll}1 & 1 & 0 & 1 \\ 0 & 1 & 0 & 0 \\ 0 & 1 & 1 & 1 \\ 0 & 0 & 1 & 1\end{array}\right]$. Then $\left(\rho \cap^{*} \tau\right) \odot T=$
$\overrightarrow{0} \neq \rho \odot T \cap^{*} \tau \odot T=\left[\begin{array}{llll}1 & 0 & 1 & 0\end{array}\right]$.

In the following, I will show what kind of $T$ is eligible to keep hold of $\rho \odot T \cap^{*} \tau \odot T=\left(\rho \cap^{*} \tau\right) \odot T$.

Definition 22. Define sum* $: 2^{U \times U} \rightarrow\left(|U|^{\downarrow}\right)^{U}$ by $\left(\operatorname{sum}^{*}(T)\right)(n):=\sum_{j \in|U| \downarrow} T(n, j)$.

Example 15. $\operatorname{sum}^{*}\left(\left[\begin{array}{lll}1 & 1 & 0 \\ 0 & 1 & 0 \\ 1 & 1 & 1\end{array}\right]\right)=\left[\begin{array}{lll}2 & 1 & 3\end{array}\right]$.

Claim 7. $\operatorname{sum}^{*}(T) \subseteq^{*} \overrightarrow{1}$ iff for all $\rho, \tau \in 2^{U}\left[\rho \odot T \cap^{*} \tau \odot T=\right.$ $\left.\left(\rho \cap^{*} \tau\right) \odot T\right]$.

Proof. Let $\rho \cap^{*} \tau=\eta$. Then $\rho \odot T \cap^{*} \tau \odot T=\left[\left(\eta \cup^{*}\left(\rho-^{*}\right.\right.\right.$ $\eta)) \odot T] \cap^{*}\left[\left(\eta \cup^{*}\left(\tau-{ }^{*} \eta\right)\right) \odot T\right]=\eta \odot T \cup^{*}\left[\left(\rho-{ }^{*} \eta\right) \odot\right.$ $\left.T \cap^{*}\left(\tau-{ }^{*} \eta\right) \odot T\right]=\eta \odot T$. On the other hand, suppose $\operatorname{sum}^{*}(T) \nsubseteq^{*} \overrightarrow{1}$, i.e., there exist $i, j, n \in|U|^{\downarrow}$ such that $i \neq j$ and $T(n, i)=T(n, j)=1$. Now define $\rho(k)=1$ if $k=$ $i$ and $\rho(k)=0$, otherwise and define $\tau(k)=1$ if $k=j$ and $\tau(k)=0$, otherwise for all $k \in|U|^{\downarrow}$. Then one has $\rho \odot T \cap^{*} \tau \odot T \neq\left(\rho \cap^{*} \tau\right) \odot T=\overrightarrow{0}$. 
Corollary 1. $\forall \rho, \tau \in 2^{U}\left[\rho \odot T \cap^{*} \tau \odot T=\left(\rho \cap^{*} \tau\right) \odot T\right]$ iff $\forall \rho \in 2^{U}\left[\rho \odot T \cap^{*}\left(\overrightarrow{1}-{ }^{*} \rho\right) \odot T=\overrightarrow{0}\right]$.

Proof. Since $\operatorname{sum}^{*}(T) \subseteq^{*} \overrightarrow{1}$ iff $\forall \rho \in 2^{U}\left[\rho \odot T \cap^{*}\left(\overrightarrow{1}-{ }^{*} \rho\right) \odot\right.$ $T=\overrightarrow{0}]$, by Claim 7 , the result follows.

Claim 8. If $\rho \subseteq^{*} \tau$, then $\rho \odot T \subseteq^{*} \tau \odot T$.

Proof. It follows immediately from the definition and Claim 3.

Example 16. Property (10) at Section 2.3 in Pawlak's paper [1] is a direct instance of this claim (via Lemma 1).

\section{Operator $-^{*}$}

Claim 9. $\left(\rho-^{*} \tau\right)^{-1}\{1\}=\rho^{-1}\{1\}-\tau^{-1}\{1\}$.

Proof. $n \in\left(\rho-^{*} \tau\right)^{-1}\{1\}$ iff $\rho(n)=1$ and $\tau(n)=0$ iff $n \in \rho^{-1}\{1\}-\tau^{-1}\{1\}$.

\section{Specification operators: po and so}

Let $\rho, \rho^{\prime}, \tau \in 2^{U}, T \in 2^{U \times U}$ and $S \subseteq 2^{U}$ be arbitrary.

Claim 10. $(\rho, T)$ is a crisp specification (defined at Definition 15) iff $(\rho \odot T) \cap^{*}\left(\left(\overrightarrow{1}-{ }^{*} \rho\right) \odot T\right)=\overrightarrow{0}$.

Proof. $\rho \odot T=\rho \odot T-{ }^{*}\left(\overrightarrow{1}-{ }^{*} \rho\right) \odot T$ iff $\rho \odot T \cap^{*}\left(\overrightarrow{1}-{ }^{*} \rho\right) \odot T=$ $\overrightarrow{0}$.

Claim 11. $s o(\rho, T) \cap^{*} s o\left(\rho^{\prime}, T\right)=\left(\rho \odot T \cap^{*} \rho^{\prime} \odot T\right)-^{*}\left(\overrightarrow{1}-{ }^{*}\right.$ $\left.\rho \cap^{*} \rho^{\prime}\right) \odot T$.

Proof. $s o(\rho, T) \cap^{*} s o\left(\rho^{\prime}, T\right)\left[\rho \odot T-^{*}\left(\overrightarrow{1}-^{*} \rho\right) \odot T\right] \cap^{*} \rho^{\prime} \odot$ $\left.T-^{*}\left(\overrightarrow{1}-{ }^{*} \rho^{\prime}\right) \odot T\right]=\left[\rho \odot T \cap^{*}\left(\rho^{\prime} \odot T-{ }^{*}\left(\overrightarrow{1}-{ }^{*} \rho^{\prime}\right) \odot T\right)\right]-^{*}$ $\left(\overrightarrow{1}-{ }^{*} \rho\right) \odot T=\left[\left(\rho \odot T \cap^{*} \rho^{\prime} \odot T-{ }^{*}\left(\overrightarrow{1}-{ }^{*} \rho^{\prime}\right) \odot T\right)\right]-{ }^{*}\left(\overrightarrow{1}-{ }^{*}\right.$ $\rho) \odot T=\rho \odot T \cap^{*} \rho^{\prime} \odot T-{ }^{*}\left[\left(\overrightarrow{1}-{ }^{*} \rho\right) \odot T \cup^{*}\left(\overrightarrow{1}-{ }^{*} \rho^{\prime}\right) \odot T\right]=$ $\rho \odot T \cap^{*} \rho^{\prime} \odot T-^{*}\left[\left(\left(\overrightarrow{1}-{ }^{*} \rho\right) \cup{ }^{*}\left(\overrightarrow{1}-{ }^{*} \rho^{\prime}\right)\right) \odot T\right]$, by Claim $6=$ $\rho \odot T \cap^{*} \rho^{\prime} \odot T-^{*}\left(\overrightarrow{1}-{ }^{*} \rho \cap^{*} \rho^{\prime}\right) \odot T$.

Claim 12. po, so are all $\subseteq^{*}$-non-decreasing functions.

Proof. Let $\rho, \tau$ be arbitrary such that $\rho \subseteq^{*} \tau$. Then one has $\rho \bullet T(x)=\sup \left\{T(x)(j): j \in \rho^{-1}(1) \leq \sup \{T(x)(j): j \in\right.$ $\left.\tau^{-1}(1)\right\}=\tau \bullet T(x)$; on the other hand, so $(\rho, T)=\rho \odot T$ - $^{*}$ $\left(\overrightarrow{1}-{ }^{*} \rho\right) \odot T \subseteq^{*} \rho \odot T-{ }^{*}\left(\overrightarrow{1}-{ }^{*} \tau\right) \odot T \subseteq^{*} \tau \odot T-{ }^{*}\left(\overrightarrow{1}-{ }^{*} \tau\right) \odot T=$ $\operatorname{so}(\tau, T)$

Corollary 2. (Monotonicity of Full Upper/Lower Bound) If $\rho \subseteq^{*} \tau$, then $l b(\rho, T) \subseteq^{*} l b(\tau, T)$ and $u b(\rho, T) \subseteq^{*} u b(\tau, T)$.

Proof. The results follow immediately from Definition 6 and 7, Claim 12, Lemma 1 and Claim 8.

Example 17. Property (10) and (11) in Pawlak's paper are such instances.

\section{MAIn RESUlts}

From Lemma 1, we know it is sufficient to study po and so in order to have best upper and lower approximations. In this section, I will present my main results in order to answer my last two questions in the section Introduction, i.e., the relations between upper and lower best approximation and the closeness of different set operations under various characteristic classifiers. We also try to find out what kind of imposition is needed to preserve all these properties. Before we go further, let us define some notions first.

Definition 23. $\{p o(\rho, T): \rho \in S\}$ is $c^{*}$-closed iff $\forall \rho \in$ $S, \exists \eta \in S$ such that $\overrightarrow{1}-{ }^{*} p o(\rho, T)=p o(\eta, T)$.

Definition 24. $\{p o(\rho, T): \rho \in S\}$ is isomorphically $c^{*}$-closed iff $\forall \rho \in S, \overrightarrow{1}-{ }^{*} p o(\rho, T)=p o\left(\overrightarrow{1}-{ }^{*} \rho, T\right)$.

Isomorphically $c^{*}$-closed is a strong relation for the complement operation. It is more informative than $c^{*}$-closed. For example, if this property holds, then one only needs to compute $p o(\rho, T)$ to in order to have a result for $p o\left(\overrightarrow{1}-{ }^{*} \rho, T\right)$. This would save a lot of resources in the process of computation and classification. It is the same for the operation so as well.

Definition 25. $\{s o(\rho, T): \rho \in S\}$ is $c^{*}$-closed iff $\forall \rho \in$ $S, \exists \eta \in S$ such that $\overrightarrow{1}-{ }^{*} s o(\rho, T)=s o(\eta, T)$.

Definition 26. $\{s o(\rho, T): \rho \in S\}$ is isomorphically $c^{*}$-closed iff $\forall \rho \in S, \overrightarrow{1}-{ }^{*} s o(\rho, T)=s o\left(\overrightarrow{1}-{ }^{*} \rho, T\right)$

Now let us define some weak relations and strong relations of the set operations $\cup^{*}$ and $\cap^{*}$ for both po and so. Again, the strong relations, i.e., isomorphically-closed operations are more informative than the weak ones.

Definition 27. $\{p o(\rho, T): \rho \in S\}$ is $\cup^{*}$-closed iff $\forall \rho, \tau \in$ $S, \exists \eta \in S$ such that $p o(\rho, T) \cup^{*} p o(\tau, T)=p o(\eta, T)$

Definition 28. $\{p o(\rho, T): \rho \in S\}$ is isomorphically $\cup^{*}$-closed iff $\forall \rho, \tau \in S, p o(\rho, T) \cup^{*} p o(\tau, T)=p o\left(\rho \cup^{*} \tau, T\right)$

When isomorphically $\cup^{*}$-closed holds, one only needs to compute $p o(\rho, T)$ and $p o(\tau, T)$ in order to compute $p o\left(\rho \cup^{*}\right.$ $\tau, T)$. In the real computation and classification, this would save a lot of resources and time. The whole argument holds for so too.

Definition 29. $\{s o(\rho, T): \rho \in S\}$ is $\cap^{*}$-closed iff $\forall \rho, \tau \in$ $S, \exists \eta \in S$ such that $s o(\rho, T) \cap^{*} s o(\tau, T)=s o(\eta, T)$

Definition 30. $\{s o(\rho, T): \rho \in S\}$ is isomorphically $\cap^{*}$-closed iff $\forall \rho, \tau \in S, s o(\rho, T) \cap^{*} s o(\tau, T)=s o\left(\rho \cap^{*} \tau, T\right)$

Definition 31. $\{p o(\rho, T): \rho \in S\}$ is $\cap^{*}$-closed iff $\forall \rho, \tau \in$ $S, \exists \eta \in S$ such that $p o(\rho, T) \cap^{*} p o(\tau, T)=p o(\eta, T)$

Definition 32. $\{p o(\rho, T): \rho \in S\}$ is isomorphically $\cap^{*}$-closed iff $\forall \rho, \tau \in S, p o(\rho, T) \cap^{*} p o(\tau, T)=p o\left(\rho \cap^{*} \tau, T\right)$

Definition 33. $\{s o(\rho, T): \rho \in S\}$ is $\cup^{*}$-closed iff $\forall \rho, \tau \in$ $S, \exists \eta \in S$ such that $s o(\rho, T) \cup^{*} s o(\tau, T)=s o(\eta, T)$ 
Definition 34. $\{s o(\rho, T): \rho \in S\}$ is isomorphically $\cup^{*}$-closed iff $\forall \rho, \tau \in S, s o(\rho, T) \cup^{*} s o(\tau, T)=s o\left(\rho \cup^{*} \tau, T\right)$.

Now let us start the derivations. Let $T \in 2^{U \times U}$ be arbitrary. Recall that sup* is already defined in Definition 16.

\section{A. Properties of unrestricted $T$}

The following result is the most general condition for a characteristic classifier $T$ as there is no special restriction imposed on $T$, i.e., $T$ could be degenerated or non-degenerated.

Theorem $1\left(\cup^{*}\right.$-Closeness of $\left.\{p o(\rho, T): \rho \in S\}\right)$. If $S \subseteq 2^{U}$ is $\cup^{*}$-closed, then $\{p o(\tau, T): \tau \in S\}$ is also $\cup^{*}$-closed.

Proof. Let $\rho, \tau \in S$ be arbitrary. Then, by Claim $6, \rho \odot T \cup^{*}$ $\tau \odot T=\left(\rho \cup^{*} \tau\right) \odot T$.

This specifies a sufficient condition that as long as a universe is closed under $\cup^{*}$ operation, po will be always closed under $\cup^{*}$ operation. An application is when one intends to design a classifying system via full rough sets and expects it is closed under union operation, then the universe that guarantees this is a universe that is closed under $\cup^{*}$ operation too.

\section{B. Properties of $T$ with $\sup ^{*}(T)=\overrightarrow{1}$}

Now we look into the non-degenerated characteristic classifier $T$. A non-degenerated characteristic classifier $T$ is a very weak requirement of $T$ that every element in the universe $U$ has some relation with others (at least itself). In other words, $T(n) \neq \overrightarrow{0}$ for all $n \in|U|^{\downarrow}$. Let $\rho, \tau \in 2^{U}$ be arbitrary. Let $T \in 2^{U \times U}$ be arbitrary such that $\sup ^{*}(T)=\overrightarrow{1}$.

Claim 13. $\overrightarrow{1}=p o(\rho, T) \cup^{*} p o\left(\overrightarrow{1}-{ }^{*} \rho, T\right)$ iff $\sup ^{*}(T)=\overrightarrow{1}$.

Proof. By Claim 6, po $(\rho, T) \cup^{*} p o\left(\overrightarrow{1}-{ }^{*} \rho, T\right)=\overrightarrow{1} \odot T$. Hence $\overrightarrow{1}=\overrightarrow{1} \odot T$ iff $\forall n \in|U|^{\downarrow}(T(n) \neq \overrightarrow{0})$, i.e., $\sup ^{*}(T)=\overrightarrow{1}$.

Claim 14. If $\sup ^{*}(T)=\overrightarrow{1}$, then $\overrightarrow{1}-{ }^{*} p o(\rho, T)=s o\left(\overrightarrow{1}-{ }^{*} \rho, T\right)$.

Proof. By Claim 13, it follows $\overrightarrow{1}-* p o(\rho, T)=\left[p o(\rho, T) \cup^{*}\right.$ $\left.p o\left(\overrightarrow{1}-{ }^{*} \rho, T\right)\right]-{ }^{*} p o(\rho, T)=p o\left(\overrightarrow{1}-{ }^{*} \rho, T\right)-{ }^{*} p o(\rho, T)$.

This is an important result as it specifies the relation between operators $p o$ and so. One can even apply it to all the special cases.

Example 18. Property (2), (3), (8) and (9) at Section 2.3 in Pawlak's paper [1] are the direct instances of this claim. With the help of other claims, property (14), (15) and (16) could also be demonstrated (via Lemma 1, Claim 14, Claim 6, Claim 13). Take property (14) for example: $u b\left(X^{*}, T\right) \cup^{*} l b(\overrightarrow{1}-*$ $\left.X^{*}, T\right)=p o\left(X^{*}, T\right) \odot T^{t} \cup^{*} s o\left(\overrightarrow{1}-{ }^{*} X^{*}\right) \odot T^{t}=p o\left(X^{*}, T\right) \odot$ $T^{t} \cup^{*} p o\left(\overrightarrow{1}-{ }^{*} X^{*}, T\right) \odot T^{t}=p o(\overrightarrow{1}, T) \odot T^{t}=\overrightarrow{1}$, where $T$ is the characteristic classifier induced by the equivalence relation.

Though the main purpose for this paper is to find out the closeness of various set operations under the specification operators $p o$ and $s o$, it is worth mentioning that some of the identities in Pawlak's paper would fail in full rough sets. For example, Property (18) holds if $T$ is the equivalencerelation-induced classifier by the fact that $T^{t}=T$ and $(\rho \odot T) \odot T^{t}=(\rho \odot T) \odot T=\rho \odot T:($ by Claim 14) $u b\left(X^{*}, T\right) \cap^{*} l b\left(\overrightarrow{1}-{ }^{*} X^{*}, T\right)$

$=\left(X^{*} \odot T\right) \odot T^{t} \cap^{*}\left(\overrightarrow{1}-{ }^{*} X^{*} \odot T\right) \odot T^{t}$

$=\left(X^{*} \odot T\right) \odot T \cap^{*}\left(\overrightarrow{1}-{ }^{*} X^{*} \odot T\right) \odot T$

$=\left(X^{*} \odot T\right) \cap^{*}\left(\overrightarrow{1}-{ }^{*} X^{*} \odot T\right)=\overrightarrow{0}$.

Incidentally, the first part of Property (4): $\overline{A p r}(\overline{A p r}(X))=$ $\overline{A p r}(X)$ is also an instance of the fact $T^{t}=T$ and $(\rho \odot T) \odot T^{t}=(\rho \odot T) \odot T=\rho \odot T$ when $T$ is the equivalencerelation-induced classifier. However, in a full relation (i.e., no restriction on the relation), this identity might fail. For example $X^{*}=\left[\begin{array}{llll}1 & 0 & 1 & 0\end{array}\right]$ and $T=\left[\begin{array}{llll}1 & 0 & 1 & 0 \\ 0 & 0 & 0 & 1 \\ 1 & 0 & 0 & 1 \\ 0 & 1 & 0 & 1\end{array}\right]$. Then $u b\left(X^{*}, T\right)=\left[\begin{array}{llll}1 & 0 & 1 & 1\end{array}\right]$ and $l b\left(\overrightarrow{1}-{ }^{*} X^{*}, T\right)=$ $\left[\begin{array}{llll}0 & 1 & 0 & 1\end{array}\right]$. Hence $u b\left(X^{*}, T\right) \cap^{*} l b\left(\overrightarrow{1}-{ }^{*} X^{*}, T\right) \neq \overrightarrow{0}$.

If $T$ is induced by a full relation, generally speaking, $(\overrightarrow{1}-*$ $p o(\rho, T)) \odot T^{t} \neq \overrightarrow{1}-{ }^{*} p o(\rho, T)$. However, if it is restricted to an equivalence relation, $\left.\left(\overrightarrow{1}-{ }^{*} p o(\rho, T)\right) \odot T^{t}=\overrightarrow{1}-{ }^{*} p o(\rho, T)\right)$ and this, together with Claim 14 (which yiels $s o(\rho, T) \odot T=$ $s o(\rho, T)$, if $T$ is an equivalence-relation-induced classifier), directly leads to validity of Property (20) and (21) in Pawlak's paper. Moreover, generally speaking, $(\rho \odot T) \odot T^{t} \neq \rho \odot T$; but, imposing a restriction with an equivalence relation leads $(\rho \odot T) \odot T^{t}=\rho \odot T$. Hence Property (5), (7), and the De Morgan's laws: (22)-(29) in Pawlak's paper are such instances. Take (5) for example:

$l b\left(l b\left(X^{*}, T\right), T\right)=\operatorname{so}\left(\operatorname{so}\left(X^{*}, T\right) \odot T^{t}, T\right) \odot T^{t}$

$=\operatorname{so}\left(\operatorname{so}\left(X^{*}, T\right), T\right)=\operatorname{so}\left(\overrightarrow{1}-{ }^{*} p o\left(\overrightarrow{1}-{ }^{*} X^{*}, T\right), T\right)$

$=\overrightarrow{1}-{ }^{*} p o\left(p o\left(\overrightarrow{1}-{ }^{*} X^{*}, T\right), T\right)=\overrightarrow{1}-{ }^{*} p o\left(\overrightarrow{1}-{ }^{*} X^{*}, T\right)$

$=s o\left(X^{*}, T\right)=s o\left(X^{*}, T\right) \odot T^{t}=l b\left(X^{*}, T\right)$.

Take (7) for example:

$l b\left(X^{*} \cap^{*} Y^{*}, T\right)=\operatorname{so}\left(X^{*} \cap^{*} Y^{*}, T\right) \odot T^{t}=s o\left(X^{*} \cap^{*} Y^{*}, T\right)$

$=\overrightarrow{1}-{ }^{*} p o\left(\overrightarrow{1}-{ }^{*}\left(X^{*} \cap^{*} Y^{*}\right), T\right)$

$=\overrightarrow{1}-{ }^{*} p o\left(\left(\overrightarrow{1}-{ }^{*} X^{*}\right) \cup^{*}\left(\overrightarrow{1}-{ }^{*} Y^{*}\right), T\right)$

$=\overrightarrow{1}-{ }^{*}\left[p o\left(\overrightarrow{1}-{ }^{*} X^{*}, T\right) \cup^{*} p o\left(\overrightarrow{1}-{ }^{*} Y^{*}, T\right)\right]$

$=\left[\overrightarrow{1}-{ }^{*} p o\left(\overrightarrow{1}-{ }^{*} X^{*}, T\right)\right] \cap^{*}\left[\overrightarrow{1}-{ }^{*} p o\left(\overrightarrow{1}-{ }^{*} Y^{*}, T\right)\right]$

$=\operatorname{so}\left(X^{*}, T\right) \cap^{*} \operatorname{so}\left(Y^{*}, T\right)$

$=s o\left(X^{*}, T\right) \odot T^{t} \cap^{*} s o\left(Y^{*}, T\right) \odot T^{t}$

$=l b\left(X^{*}, T\right) \cap^{*} l b\left(Y^{*}, T\right)$.

Theorem 2 (Closeness: $\left(\{p o(\rho, T): \rho \in S\}, c^{*}\right)$ and $\left.\left(\{\operatorname{so}(\rho, T): \rho \in S\}, c^{*}\right)\right)$. If $\sup ^{*}(T)=\overrightarrow{1}$ and $S \subseteq 2^{U}$ is $c^{*}$-closed, then $\{p o(\rho, T): \rho \in S\}$ is $c^{*}-$ closed iff $\{s o(\rho, T): \rho \in S\}$ is $c^{*}-$ closed.

Proof. Let $\rho \in S$ be arbitrary. By Claim 14, we have $\overrightarrow{1}-^{*}$ $\rho \odot T=\operatorname{so}\left(\overrightarrow{1}-{ }^{*} \rho, T\right)=\overrightarrow{1}-{ }^{*}\left[\overrightarrow{1}-{ }^{*} s o\left(\overrightarrow{1}-{ }^{*} \rho, T\right)\right]=\overrightarrow{1}-{ }^{*}$ so $(\eta, T)=\left(\overrightarrow{1}-^{*} \eta\right) \odot T$ for some $\eta \in S$; similarly, $\overrightarrow{1}-^{*}$ $s o(\rho, T)=\overrightarrow{1}-^{*}\left[\overrightarrow{1}-^{*} p o\left(\overrightarrow{1}-{ }^{*} \rho, T\right)\right]=\overrightarrow{1}-{ }^{*} p o(\delta, T)=$ so $\left(\overrightarrow{1}-{ }^{*} \delta, T\right)$ for some $\delta \in S$.

This theorem says that for any non-degenerated characteristic classifier and a $c^{*}$-closed universe (or subset), the operators po and so behaves identically with respect to the closeness of $c^{*}$ operation. This is a good feature, since it simplifies some derivations when one tries to find out the $c^{*}$-closeness. $\mathrm{He}$ then only need to check one of the two operators. 
Theorem 3. If $\sup ^{*}(T)=\overrightarrow{1}$ and $S \subseteq 2^{U}$ is $c *$-closed, then $\{s o(\rho, T): \rho \in S\}$ is isomorphically $c^{*}$-closed iff $\{p o(\rho, T)$ : $\rho \in S\}$ is isomorphically $c^{*}$-closed.

Proof. Let $\rho \in S$ be arbitrary. By Claim 14, $\overrightarrow{1}-{ }^{*} p o(\rho, T)=$ $s o\left(\overrightarrow{1}-{ }^{*} \rho, T\right)=\overrightarrow{1}-^{*} s o(\rho, T)=p o\left(\overrightarrow{1}-{ }^{*} \rho, T\right)$; similarly, $\overrightarrow{1}-{ }^{*} \operatorname{so}(\rho, T)=\operatorname{po}\left(\overrightarrow{1}-{ }^{*} \rho, T\right)=\overrightarrow{1}-{ }^{*} \operatorname{po}(\rho, T)=\operatorname{so}\left(\overrightarrow{1}-{ }^{*}\right.$ $\rho, T)$.

Theorem 4 (Closeness: $\left(\{s o(\rho, T): \quad \rho \in S\}, \cap^{*}\right)$ and $\left.\left(\{p o(\rho, T: \rho \in S)\}, \cup^{*}\right)\right)$. If $\sup ^{*}(T)=\overrightarrow{1}$ and $S \subseteq 2^{U}$ is $c *$-closed, then $\{s o(\tau, T): \tau \in S\}$ is $\cap^{*}$-close iff $\{p o(\tau, T): \tau \in S\}$ is $\cup^{*}$-closed.

Proof. Let $\rho, \tau \in S$ be arbitrary. By Claim 14, po $(\rho, T) \cup^{*}$ $p o(\tau, T)=\overrightarrow{1}-^{*}\left[\operatorname{so}\left(\overrightarrow{1}-{ }^{*} \rho, T\right) \cap^{*} \operatorname{so}\left(\overrightarrow{1}-{ }^{*} \tau, T\right)\right]=\overrightarrow{1}-{ }^{*}$ $s o(\eta, T)=p o\left(\overrightarrow{1}-^{*} \eta, T\right)$ for some $\eta \in S$. Similarly, $s o(\rho, T) \cap^{*} s o(\tau, T)=\overrightarrow{1}-{ }^{*}\left[p o\left(\overrightarrow{1}-{ }^{*} \rho, T\right) \cup^{*} p o\left(\overrightarrow{1}-{ }^{*} \tau, T\right)\right]=$ $\overrightarrow{1}-{ }^{*} p o(\delta, T)=\operatorname{so}\left(\overrightarrow{1}-{ }^{*} \delta, T\right)$ for some $\delta \in S$.

Theorem 5. If $\sup ^{*}(T)=\overrightarrow{1}$ and $S \subseteq 2^{U}$ is $c *$-closed, then $\{s o(\rho, T): \rho \in S\}$ is isomorphically $\cap^{*}$-closed iff $\{p o(\rho, T)$ : $\rho \in S\}$ is isomorphically $\cup^{*}$-closed.

Proof. Let $\rho, \tau \in S$ be arbitrary. By Claim 14, po $(\rho, T) \cup *$ $\operatorname{po}(\tau, T)=\overrightarrow{1}-{ }^{*}\left[\operatorname{so}\left(\overrightarrow{1}-{ }^{*} \rho, T\right) \cap^{*} \operatorname{so}\left(\overrightarrow{1}-{ }^{*} \tau, T\right)\right]=\overrightarrow{1}-{ }^{*} \operatorname{so}\left(\left(\overrightarrow{1}-{ }^{*}\right.\right.$ $\left.\rho) \cap^{*}\left(\overrightarrow{1}-{ }^{*} \tau\right), T\right)=\overrightarrow{1}-{ }^{*} s o\left(\overrightarrow{1}-{ }^{*} \rho \cup^{*} \tau, T\right)=p o\left(\rho \cup^{*} \tau, T\right)$. Similarly, $s o(\rho, T) \cap^{*} s o(\tau, T)=\overrightarrow{1}-{ }^{*}\left[p o\left(\overrightarrow{1}-{ }^{*} \rho, T\right) \cup^{*} p o\left(\overrightarrow{1}-{ }^{*}\right.\right.$ $\tau, T)]=\overrightarrow{1}-{ }^{*} p o\left(\overrightarrow{1}-{ }^{*} \rho \cap^{*} \tau, T\right)=\operatorname{so}\left(\rho \cap^{*} \tau, T\right)$.

Corollary 3. If $\sup ^{*}(T)=\overrightarrow{1}$ and $S \subseteq 2^{U}$ is $\cup^{*}$-closed, $\cap^{*}$-closed and $c^{*}$-closed, then $\{s o(\rho, T): \rho \in S\}$ is isomorphically $\cap^{*}$-closed.

Proof. From Claim 6, $\{p o(\rho, T): \rho \in S\}$ is isomorphically $\cup^{*}$-closed and thus by this theorem, the result follows.

Theorem 4 and 5 show the relation between $(\{p o(\rho, T)$ : $\left.\rho \in S\}, \cup^{*}\right)$ and $\left(\{s o(\rho, T): \rho \in S\}, \cap^{*}\right)$. This again simplifies our derivations or computations since we only have to check or compute either one of the two operators. It is also useful when one tries to find a universe that will keep the relations hold, he only needs to consider one operator.

Claim 15. $s o(\rho, T) \cup^{*} s o(\tau, T)=\overrightarrow{1}-{ }^{*}\left[p o\left(\overrightarrow{1}-{ }^{*} \rho, T\right) \cap^{*}\right.$ $\left.p o\left(\overrightarrow{1}-{ }^{*} \tau, T\right)\right]$.

Proof. By Claim 14, it follows $s o(\rho, T) \cup^{*} s o(\tau, T)=s o(\overrightarrow{1}-$ $(\overrightarrow{1}-\rho), T) \cup^{*} s o(\overrightarrow{1}-(\overrightarrow{1}-\tau), T)=\left[\overrightarrow{1}-{ }^{*} p o\left(\overrightarrow{1}-{ }^{*} \rho, T\right)\right] \cup *[\overrightarrow{1}-*$ $\left.p o\left(\overrightarrow{1}-^{*} \tau, T\right)\right]=\overrightarrow{1}-^{*}\left[p o\left(\overrightarrow{1}-{ }^{*} \rho, T\right) \cap^{*} \operatorname{po}\left(\overrightarrow{1}-{ }^{*} \tau, T\right)\right]$.

Example 19. Property (22) at Section 2.3 in Pawlak's paper [1] is a direct instance of this claim (via Lemma 1).

Claim 16. $p o(\rho, T) \cap^{*} p o(\tau, T)=\overrightarrow{1}-^{*}\left[s o\left(\overrightarrow{1}-{ }^{*} \rho, T\right) \cup^{*}\right.$ so $\left.\left(\overrightarrow{1}-{ }^{*} \tau, T\right)\right]$.

Proof. By Claim 14, it follows $p o(\rho, T) \cap^{*} \operatorname{po}(\tau, T)=\left[\overrightarrow{1}-^{*}\right.$ $\left.\operatorname{so}\left(\overrightarrow{1}-{ }^{*} \rho, T\right)\right] \cap *\left[\overrightarrow{1}-{ }^{*} \operatorname{so}\left(\overrightarrow{1}-{ }^{*} \tau, T\right)\right]=\overrightarrow{1}-{ }^{*}\left[\operatorname{so}\left(\overrightarrow{1}-{ }^{*} \rho, T\right) \cup^{*}\right.$ so $\left.\left(\overrightarrow{1}-{ }^{*} \tau, T\right)\right]$.

Theorem 6 (Closeness: $\left(\{s o(\rho, T): \rho \in S\}, \cup^{*}\right)$ and $\left.\left(\{p o(\rho, T): \rho \in S\}, \cap^{*}\right)\right)$. If $\sup ^{*}(T)=\overrightarrow{1}$ and $S \subseteq 2^{U}$ is $c *$-closed, then $\{s o(\rho, T): \rho \in S\}$ is $\cup^{*}$-closed iff $\{p o(\rho, T): \rho \in S\}$ is $\cap^{*}$-closed.

Proof. Let $\rho, \tau \in S$ be arbitrary. By Claim 16, po $(\rho, T) \cap^{*}$ $p o(\tau, T)=\overrightarrow{1}-^{*}\left[\operatorname{so}\left(\overrightarrow{1}-{ }^{*} \rho, T\right) \cup^{*} \operatorname{so}\left(\overrightarrow{1}-{ }^{*} \tau, T\right)\right]=\overrightarrow{1}-{ }^{*}$ $s o(\eta, T)=p o\left(\overrightarrow{1}-^{*} \eta, T\right)$ for some $\eta \in S$, by Claim 14 ; similarly, by Claim 15 , so $(\rho, T) \cup * s o(\tau, T)=\overrightarrow{1}-{ }^{*}\left[p o\left(\overrightarrow{1}-{ }^{*}\right.\right.$ $\left.\rho, T) \cap^{*} p o\left(\overrightarrow{1}-{ }^{*} \tau, T\right)\right]=\overrightarrow{1}-^{*} p o(\delta, T)=s o\left(\overrightarrow{1}-{ }^{*} \delta, T\right)$ for some $\delta \in S$, by Claim 14 .

Theorem 6 and the following 7 show the relation between $\left(\{p o(\rho, T): \rho \in S\}, \cap^{*}\right)$ and $\left(\{s o(\rho, T): \rho \in S\}, \cup^{*}\right)$. As analyzed previously, this again simplifies our computations since we only have to check one of the two operators $p 0$ and so. It is also useful when one tries to construct or design a universe that will keep these relations hold, he only has to consider one operator instead.

Theorem 7. If $\sup ^{*}(T)=\overrightarrow{1}$ and $S \subseteq 2^{U}$ is $c *$-closed, then $\{\operatorname{so}(\rho, T): \rho \in S\}$ is isomorphically $\cup^{*}$-closed iff $\{p o(\rho, T)$ : $\rho \in S\}$ is isomorphically $\cap^{*}$-closed.

Proof. Let $\rho, \tau \in S$ be arbitrary. By Claim 14, 15 and 16, it follows $p o(\rho, T) \cap * \operatorname{po}(\tau, T)=\overrightarrow{1}-{ }^{*}\left[\operatorname{so}\left(\overrightarrow{1}-{ }^{*} \rho, T\right) \cup^{*} s o\left(\overrightarrow{1}-{ }^{*}\right.\right.$ $\tau, T)]=\overrightarrow{1}-{ }^{*} \operatorname{so}\left(\left(\overrightarrow{1}-{ }^{*} \rho\right) \cup^{*}\left(\overrightarrow{1}-{ }^{*} \tau\right), T\right)=\overrightarrow{1}-{ }^{*} \operatorname{so}\left(\overrightarrow{1}-{ }^{*}\right.$ $\left.\rho \cap^{*} \tau, T\right)=p o\left(\rho \cap^{*} \tau, T\right)$. Similarly, so $(\rho, T) \cup^{*} \operatorname{so}(\tau, T)=$ $\overrightarrow{1}-{ }^{*}\left[p o\left(\overrightarrow{1}-{ }^{*} \rho, T\right) \cap \cap^{*} p o\left(\overrightarrow{1}-{ }^{*} \tau, T\right)\right]=\overrightarrow{1}-{ }^{*} p o\left(\overrightarrow{1}-{ }^{*} \rho \cup^{*} \tau, T\right)=$ $\operatorname{so}\left(\rho \cup^{*} \tau, T\right)$.

\section{CONCLUSIONS AND FUture WORK}

I provide an approach to analyze full rough sets, i.e., the usual rough sets, but with an arbitrary binary relation. The framework for this generalization offers a different perspective to look into Pawlak's rough sets. In this paper, I have analyzed and extended Pawlak's approach for characterization and defined several operators to accommodate such extension. I define the set specifications by two operators: probable operator and sure operator. These operators serve as logical conditions. I then study the properties of these logical operations. These operators also offer some computational advantages. I also study the properties of the closeness of set operations regarding full rough sets and compare their differences with Pawlak's rough sets. With this approach, one would be able to construct more complicated classifiers based on arbitrary relations. This could also enable one to define a classifier based on his own design or comprehension. For further research, one can extend the usual $\cup$-operation when defining a rough set. Since we have already separated the set specifications from such operation and known the properties of these set specifications, the introduction of other alternative of $\cup$-operation becomes feasible.

\section{REFERENCES}

[1] Zdzislaw Pawlak, "Rough Sets," International Journal of Computer and Information Sciences, Vol. 11, No. 5, 1982

[2] Zdzislaw Pawlak, "Rough Sets: theoretical aspects of reasoning about data," Springer Science+Business Media Dordrecht, 1991.

[3] Y.Y. Yao, "On generalizing rough set theory", Rough Sets, Fuzzy Sets, Data Mining, and Granular Computing, Proceedings of the 9th International Conference (RSFDGrC 2003), LNAI 2639, pp. 44-51, 2003. 
[4] Y,Y, Yao and S.K.M. Wong: http://www2.cs.uregina.ca/ yyao/PAPERS/relation.pdf.

[5] Y.Y. Yao, "Generalized rough set models" Rough Sets in Knowledge Discovery, Polkowski, L. and Skowron, A. (Eds.), Physica-Verlag, Heidelberg, pp. 286-318, 1998.

[6] Y.Y. Yao and T.Y. Lin, "Generalization of rough sets using modal logic,", Intelligent Automation and Soft Computing, An International Journal,
Vol. 2, No. 2, pp. 103-120, 1996.

[7] Hung Son Nguyen and Andrzej Skowron, "Rough Sets: From Rudiment to Challenges," Rough Sets and Intelligent Systems Professor Zdzisaw Pawlak in Memoriam, Vol. 1, Chapter 3, Springer-Verlag Berlin Heidelberg 2013.

[8] Lotfi A. Zaden, "Fuzzy Sets", Information and Control 8, 338-353, 1965. 